\title{
Normal coronary angiograms: financial victory from the brink of clinical defeat?
}

\author{
Bernard Keavney, Yasser M Haider, Alistair J McCance, J Douglas Skehan
}

\begin{abstract}
Objective-To examine the hypothesis that, in patients undergoing coronary angiography for suspected ischaemic heart disease, a normal angiographic result is associated with a fall in consumption of health care resources following the angiogram.

Design-Retrospective cost-benefit analysis comparing the 12 month periods before and after coronary angiography.

Setting-Tertiary cardiac referral centre. Subjects -69 consecutive patients investigated in the financial year 1991-92 whose angiograms were normal.

Main outcome measures-Drug and hospital admission costs in the 12 month periods before and after angiography; urgent and elective consultations with general practitioner in that time.

Results-The mean cost of care per patient in the year before investigation was $£ 656 \cdot 89$. A highly significant fall in all indices of resource consumption was observed in the year following investigation, the mean resulting difference in the cost of care being $£ 35.15$ per month. The cost of coronary angiography would, if this fall were maintained, be recouped in a mean time of 18 months.

Conclusions-Patients suspected on clinical grounds to have coronary atherosclerosis who are found at angiography to have normal coronary arteries are heavy consumers of health care resources. Early investigation for these patients is safe and has beneficial resource consequences in the medium term.
\end{abstract}

(Heart 1996;75:623-625)

Department of Cardiology, Glenfield General Hospital, Groby Road, Leicester, United Kingdom

B Keavney

Y M M Haider

A J McCance

J D Skehan

Correspondence to:

Dr Bernard Keavney,
Wellcome Trust Centre for

Wellcome Trust Centre for

Human Genetics, Windmill

OX3 7BN, UK.

Accepted for publication

6 December 1995 erosclerosis on the grounds of history, examination, and non-invasive testing who are found at angiography to have normal coronary anatomy are commonly encountered; rates for normal coronary angiograms vary from $5 \%$ to $30 \%$ of all diagnostic angiograms in published series. ${ }^{12}$ Patients with normal coronary angiograms under these circumstances are known to have a good prognosis in mortality terms, with 7 to 10 year survival rates of approximately $98 \% .{ }^{34}$ However, considerable morbidity and disability persist even after a normal coronary angiogram in many patients, whose course is characterised by continuing inability to work, recurrent admissions to hospital for chest pain, and ongoing use of cardiac drugs. $^{56}$ These patients will continue to require medical attention, investigation for other causes of pain (the commonest differential diagnoses being oesophageal dysfunction and panic disorder), and possibly drug treatment in the long term. They will therefore continue to consume health care resources, but whether consumption is at the same rate as before angiography is unknown.

A normal coronary angiogram is sometimes interpreted as a failure of pre-procedure evaluation, an unnecessary risk to the patient, and a waste of scarce resources. It is possible, however, that the diagnostic precision afforded by knowledge of the coronary anatomy has benefits in terms of illness behaviour and health care resource consumption that far outweigh the one-off cost of the investigation, provided it can be shown to be safe.

For example, in the case of patients with "chest pain, query cause", subsequently diagnosed as suffering from panic disorder, it has been suggested that cardiac catheterisation early in the course of the illness may not only aid medical attendants in reducing the number of repeat hospital admissions and cost of drugs, but also avoid the illness behaviour "taking root", whereupon patients are less willing to accept reassurance that their pain is of non-cardiac origin. ${ }^{7}$

We have therefore carried out a cost-benefit analysis to determine whether a normal coronary angiogram is associated with a fall in consumption of health care resources in the year following angiography compared with the year before angiography, in a retrospective study of patients referred with suspected coronary artery disease to the Regional Cardiothoracic Centre serving South Trent (population catchment 830 000).

\section{Methods}

Sixty nine patients undergoing diagnostic coronary angiography for angina-like chest pain during the financial year 1 April 1991 to 
31 March 1992 whose left ventricular and coronary angiograms were normal were identified, and their hospital notes screened. Data retrieved from the hospital notes included age, sex, drugs taken for chest pain, number of nights spent in hospital for the procedure, result of pre-angiography exercise test, and presence of cardiac risk factors.

Referring general practitioners (GPs) were surveyed by questionnaire. GPs were asked to list the number of emergency callouts and acute hospital admissions for chest pain in the year before and the year following cardiac catheterisation, the number of routine consultations for chest pain in that time, and the patient's current treatment for chest pain. GPs were also asked whether their management of the patient had become easier as a result of the angiogram.

The cost of medicines was calculated from the British national formulary, 21 st edition. The cost of a diagnostic coronary angiogram was estimated as the contract price for coronary angiography at our hospital in the financial year 1992-1993, no figures for the period in question being available. The cost of a hospital admission for chest pain was estimated to be the cost of one night's stay in the cardiac high dependency unit.

Pre and post procedure costs, consultation rates, and hospital admission rates were compared using the Wilcoxson signed rank test.

\section{Results}

The 69 patients selected for study represented $8 \%$ of the diagnostic catheter workload in the period of study. The median age of patients was 54 years, and $66 \%$ were female. Preangiography exercise tests in the hospital notes were reviewed, and interpreted as positive in 34 patients ( $49 \%$ ), and negative in 17 patients $(25 \%)$. No exercise test was available for review in 18 patients (26\%). There was no documented procedure-related morbidity or mortality. There was one death in the group of 69 patients in the year after angiography; this

Table 1 Changes in resource consumption in years before and after angiography. Values are means (SEM)

\begin{tabular}{llll}
\hline Variable & Year before angiography & Year after angiography & P value \\
\hline Cost of drugs per month $(£)$ & $11.44(1 \cdot 12)$ & $6.59(1 \cdot 16)$ & 0.0004 \\
Acute admissions/patient & $0.91(0.25)$ & $0.27(0 \cdot 1)$ & 0.0232 \\
GP consultations/patient & $4.86(0.88)$ & $3.143(0.82)$ & 0.0002 \\
GP urgent calls/patient & $1.523(0.4)$ & $0.609(0.21)$ & 0.024 \\
\hline
\end{tabular}

Table 2 Cost-benefit analysis

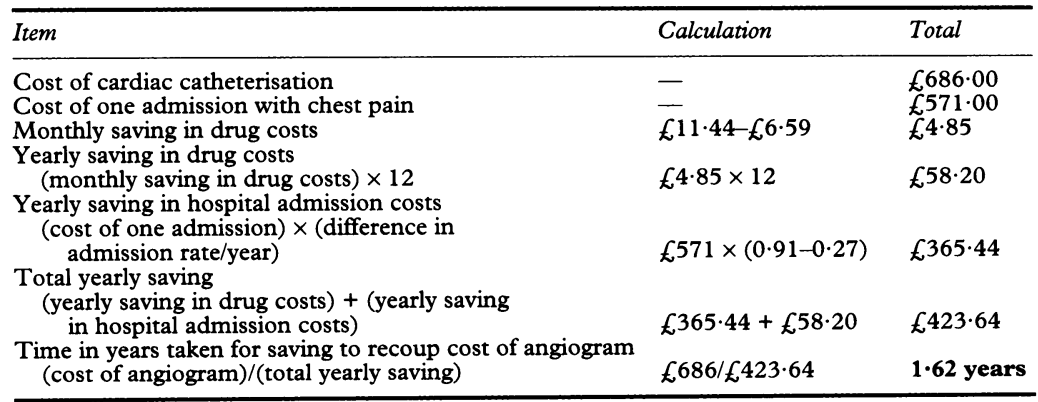

was sudden, and no necropsy data were available.

Drug cost, hospital admission, and consultation data are summarised in Table 1. Statistically significant falls in monthly drug costs, acute admissions to hospital, routine GP consultations, and urgent GP callouts in the year following angiography compared to the year preceding angiography were observed. Of the responding GPs, $53 \%$ said that the angiogram had made their management of the patient easier; the remainder felt the management had not changed in difficulty.

In the financial year 1992-1993, the contract price at our hospital for a diagnostic cardiac catheterisation was $£ 686$; we have used this figure as the cost of a diagnostic angiogram for the purposes of cost analysis. The figure takes into account necessary precatheter workup, catheter laboratory time, and cost of disposables, together with some allowance for overnight or longer stay in that proportion of patients who are not day cases, or who suffer a complication. All our cases were uncomplicated, and the great majority performed as day cases, so this figure probably represents an upper estimate of the cost to the health service of the procedure in these patients.

Our estimate of the time taken for the angiogram to "pay for itself" will therefore be conservative.

Fundholding GPs are not charged for emergency admissions, but to estimate the cost of an emergency admission with chest pain, we considered the charge made for one night on the cardiac high dependency unit $(£, 571)$ an appropriate figure. In doing so, we anticipated a "best case" scenario, whereby the patient would stay overnight, have a cardiac enzyme test together with two serial ECGs at admission and 12-24 hours afterwards to exclude myocardial infarction, and be discharged the following day. We did not include the cost of ambulance transport, other blood or radiological tests, or subsequent outpatient follow up. We considered that these assumptions would produce the most stringent test for our hypothesis.

The cost-benefit analysis is presented in table 2. The time taken for the cost of coronary angiography to be recouped was calculated by dividing the cost of an angiogram by the annual difference in mean medication and hospital admission costs before and after the procedure. The figure obtained was 1.62 years. This excludes the cost-benefit of reduced GP consultations, both routine and urgent, following the catheterisation, which were not costed. The majority of the cost-benefit lies in the reduced admission rate for these patients in the year following catheterisation.

\section{Discussion}

This cost-benefit study of coronary angiography in patients referred to a tertiary centre and suspected on clinical grounds of having coronary artery disease indicates that, following a normal result, the cost of patients' medica- 
tions, requirement for urgent or elective consultation with their GP, and need for urgent hospital admission all fall in the succeeding year. We calculate that the cost of the catheterisation would be recouped in just over 18 months if the trends we have observed in the first year persist. We have also confirmed the safety of the procedure, with no morbidity or mortality occurring as a result of catheterisation, although our sample size is insufficiently large to draw quantitative conclusions on this issue. Normal findings occurred in $8 \%$ of diagnostic catheterisations during the time period studied-comparable with previously published studies-suggesting that selection criteria for coronary angiography in our unit are adequate. It is of interest that only $53 \%$ of GPs had found the arteriogram had made the patient's management easier for them, emphasising the continued morbidity and symptoms suffered by these patients.

Both cardiac and non-cardiac syndromes may cause chest pain with normal coronary angiograms. Patients with structural heart disease other than in the coronary arteries that may lead to angina (such as mitral valve prolapse, aortic valve disease, and hypertrophic cardiomyopathy) have been excluded from the current study. The term syndrome $\mathrm{X}$ (anginalike chest pain, a positive exercise test, and angiographically normal coronary arteries) would be applicable to $49 \%$ of our subjects; the aetiology of this syndrome is the subject of some controversy ${ }^{89}$ and will not be further discussed. In keeping with practice in most catheterisation laboratories, we do not routinely pursue further investigation of the coronary arteries following a normal angiogram.

Non-cardiac syndromes such as oesophageal abnormalities and primary psychiatric disturbances, most notably panic disorder, have commonly been found in patients with normal coronary arteries and chest pain, with one or other being present in $30-50 \%$ of cases. ${ }^{1011}$ This study, in line with clinical activity in the unit, does not include systematic investigation for these non-cardiac syndromes. Further investigation for the presence of these disorders is generally directed by the GP or primary physician. Our questionnaire asked if further investigations for chest pain had been pursued; in $75 \%$ of cases they had not been. We believe this indicates that the exclusion of obstructive coronary disease is of major benefit to the GP or primary physician in the management of the patient.

To what extent are our results generalisable to patients presenting to their GP with unexplained chest pain? The patients we have studied were a relatively highly selected group: clinical evaluation and non-invasive testing had determined that the prior probability of disease in the cohort was at least moderate, and Bayes' theorem would therefore predict that the value of the diagnostic test would be maximised in such a group. If angiography were indiscriminately applied, it is highly probable that there would be unnecessary morbidity and adverse cost outcomes. In no sense, therefore, can our results be interpreted as advocating angiography as a substitute for careful clinical and non-invasive evaluation.

\section{CONCLUSIONS}

In this study, cardiac catheterisation proved to be a safe and cost-effective investigation in patients investigated for occlusive coronary disease whose coronary arteriograms were normal. We have confirmed that these patients are commonly encountered in cardiological practice, and that the cost of caring for them is high. We have shown that the use of angiography in patients with a reasonable clinical likelihood of coronary disease, particularly those requiring multiple anti-anginal drugs and repeated consultations, has significant cost benefits in the medium term in addition to the clinical benefits of knowing the coronary anatomy, and is therefore to be recommended at an early stage. A normal result does not mean that the test has been improperly applied.

We thank consultant colleagues for permission to study their patients, and all the participating general practitioners.

1 Cannon RO. Chest pain with normal coronary angiograms. Editorial. N Engl $\mathfrak{f}$ Med 1993;328:1706-8.

2 Proudfit WL, Shirey EK, Sones FM. Selective cine coronary arteriography, correlation with clinical findings in 1000 patients. Circulation 1966;33:901-10.

3 Kemp HG, Kramal RA, Vlietstra RE, et al. Seven year survival of patients with normal or near normal coronary arteriograms: A CASS registry study. $\mathcal{f} \mathrm{Am}$ Coll Cardio 1986;7:479-83

4 Papanicolaou MN, Califf RM, Hlatky MA, et al. Prognostic implications of angiographically normal and insignificantly narrowed coronary arteries. $\mathrm{Am} \mathcal{F}$ Cardiol 1986;58:1181-7.

5 Ockene IS, Shay MJ, Alpert JS, et al. Unexplained chest pain in patients with normal coronary arteriograms-a follow-up study of functional status. $N$ Engl $\mathcal{F}$ Med 1980 ; 303:1249-52.

6 Lavey EB, Winkle RA. Continuing disability of patients with chest pain and normal coronary arteriograms. Chron Dis 1978;32:191-6.

7 Assey ME. The puzzle of normal coronary arteries in the patient with chest pain: what to do? Clin Cardiol 1993;16: 170-80.

8 Camm RO, Watson RM, Rosing DR, Epstein SE. Angina caused by reduced vasodilator reserve of the small coronary arteries. $\mathcal{F}$ Am Coll Cardiol 1983;6:1359-73.

9 Camici PG, Gistri R, Lorenzoni R, et al. Coronary reserve and exercise ECG in patients with chest pain and normal coronary angiograms. Circulation 1992;86:179-86.

10 Svensson O, Stenport G, Tibblung L, Wranne B. Oesophageal function and coronary angiogram in pesophageal function and coronary angiogram in 204:173-8.

11 Beitman BD. Panic disorder in patients with angiographically normal coronary arteries. Am $f$ Med 1992;92. $33-40 S$. 